\title{
Is the Chemical Strategy for Imbuing "Polyene" Character in Diketopyrrolopyrrole-Based Chromophores Sufficient for Singlet Fission?
}

Tushita Mukhopadhyay, ${ }^{\dagger, \S}$ Andrew J. Musser, ${ }^{*, \perp, \downarrow, \S \odot ~}$ Boregowda Puttaraju, $^{\dagger}$ Joydeep Dhar, $^{\dagger}$ Richard H. Friend, ${ }^{\ddagger}$ and Satish Patil ${ }^{*} \dagger$

${ }^{\dagger}$ Solid State and Structural Chemistry Unit, Indian Institute of Science, Bangalore 560012, India

${ }^{\perp}$ Department of Physics and Astronomy, University of Sheffield, Sheffield, United Kingdom

${ }^{\ddagger}$ Cavendish Laboratory, Department of Physics, University of Cambridge, Cambridge CB3 0HE, United Kingdom

\section{Supporting Information}

ABSTRACT: In this work, we have rationally designed and synthesized a novel thiophene-diketopyrrolopyrrole (TDPP)-vinyl-based dimer. We have investigated the optical and electronic properties and have probed the photophysical dynamics using transient absorption to investigate the possibility of singlet exciton fission. These revealed extremely rapid decay to the ground state $(<50 \mathrm{ps})$, which we confirm is due to intramolecular excitonic processes rather than large-scale conformational change enabled by the vinyl linker. In all cases, the main excited state appears to be "dark", suggesting rapid internal conversion into a dark $2 \mathrm{~A}_{\mathrm{g}}$-type singlet state. We found no evidence of triplet formation in TDPP-V-TDPP under direct photoexcitation. This may be a consequence of significant singlet stabilization in the dimer, bringing it below the energy needed to form two triplets. Our studies on this model compound set valuable lessons for design of novel triplet-forming materials and highlight the need for more broadly applicable design principles.

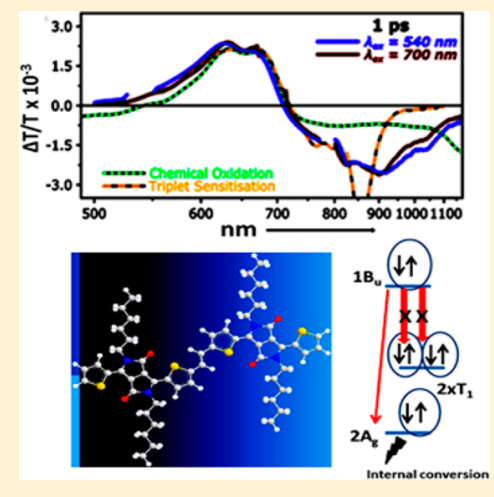

onsiderable efforts have been directed toward the development of efficient excitonic solar cells based on organic materials, through a multitude of advancements in the design of appropriate molecular backbones, thin-film processing conditions, and device-architectural engineering. ${ }^{1-5}$ To date, the highest power conversion efficiency achieved in organic solar cells is in the range of $\sim 10 \%{ }^{6}$ One critical limitation for these and indeed all other single-junction solar cell technologies arises from fundamental thermodynamic processes such as relaxation of high-energy carriers/excitons to the band edge. The consideration of such processes yields the ShockleyQuiesser efficiency limit of $\sim 33 \%$ for single-junction solar cells. $^{7,8}$ One of the ways to circumvent this limitation is by invoking and implementing the concept of singlet exciton fission (SEF). 8 This process involves the conversion of a singlet exciton into a pair of low-energy triplet excitons, which are initially coupled into an overall singlet state. This maintenance of singlet spin enables SEF to proceed on ultrafast time scales to outcompete other loss channels. ${ }^{10}$

Efficient SEF relies on correct alignment of the singlet and triplet energy states, such that $2 E\left(T_{1}\right) \leq E\left(S_{1}\right) .{ }^{11}$ By thereby splitting a high-energy exciton into two low-energy excitons, it becomes possible to harvest a significant proportion of the energy in "visible spectrum" photons typically lost to thermalization, resulting in a new maximum efficiency limit of $\sim 44 \%{ }^{12}$ In recent years, computational and experimental studies have demonstrated that acenes, for example, pentacene, exhibit efficient SEF with $\sim 200 \%$ triplet yield on time scales as fast as 80 fs. ${ }^{13-19}$ SEF proceeds either through an intermolecular or intramolecular mechanism. ${ }^{20-24}$ For both mechanisms, SEF rates and efficiencies can be controlled by fine tuning the strength of interchromophore coupling, which in the case of intermolecular fission entails the difficult task of optimizing chromophore packing within the film. ${ }^{18,24,25}$ Furthermore, several groups have demonstrated that coupling between singlet and triplet pair manifolds can be very effectively mediated by intermediate charge-transfer (CT) states. $^{21-23,26}$ This insight provides design criteria for the selection of building blocks for new intramolecular SEF materials that satisfy two key requirements for efficient $S E F$, an increased $S_{1}-T_{1}$ gap (to achieve $\left.2 \times E\left(\mathrm{~T}_{1}\right) \leq E\left(\mathrm{~S}_{1}\right)\right)$ and a lowest-lying optical excitation with significant $\mathrm{CT}$ character that can mediate the SEF process. $^{27-31}$

There is little knowledge about the degree of interchromophore coupling needed to make SEF competitive with fluorescence and to yet allow the component triplets of the singlet-coupled triplet pair to "escape" and behave as independent excitations. $^{10,32,33}$ It is evident, however, that these processes require a delicate balance of chemical design parameters. These processes depend largely on the crystal

Received: December 12, 2016

Accepted: January 23, 2017

Published: January 23, 2017 


\section{Scheme 1. Synthesis of TDPP-V-TDPP}
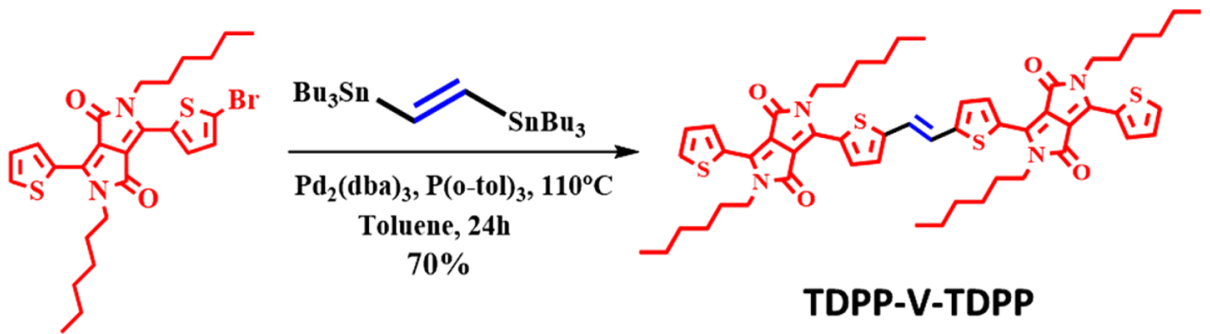

environment, whether by positioning the molecules favorably for direct interaction or by stabilizing the low-energy CT states that serve as virtual states in the coupling process. ${ }^{26,34}$ The interchromophore interaction can range from mere physical contact through indirect covalent coupling to direct covalent bonding that permits strong $\pi$-conjugation. The extent of coupling has direct effects on the SEF rate but also indirectly affects the process by modifying the energy of the states of the isolated monomers and thus the exoergicity of SEF. ${ }^{33,35}$ This interchromophore coupling can be controlled by tailoring the chemical structure. ${ }^{10,18,24,36-40}$ One rational design approach to optimize this coupling for intramolecular SEF materials is based on alternately coupling electron-rich and electron-deficient moieties to form a quinoidal $\mathrm{D}-\mathrm{A}-\mathrm{D}$ backbone imparting significant CT character to the lowest-energy optical excitation. ${ }^{18,26,32,41}$ The resulting CT state possesses a large absorption cross section and may couple more strongly to multiexciton triplet states than pure singlet Frenkel excitons. $^{27,42}$ We highlight in particular the design strategy proposed with this motif for conjugated polymers and oligomers. In that work, the oxidized thiophene acceptor found to be necessary to enable SEF simultaneously endows materials with significant oligoene/polyene character, suggesting that a judicious balance of $\mathrm{CT}$ and polyene character is needed. ${ }^{26,43}$

Indeed, the property of intramolecular SEF can also be achieved by incorporating oligoene-like moieties. ${ }^{4,45}$ In conventional polyenes, the lowest $2 \mathrm{~A}_{\mathrm{g}}$ states lie below the $1 B_{u}$ states, enabling rapid (few ps) internal conversion to this "dark" singlet that renders them largely nonemissive. It has long been considered that the $2 \mathrm{~A}_{\mathrm{g}}$ state is crucial to enable SEF as it can be alternatively described as a bound triplet pair. ${ }^{10,46}$ However, more recent experimental evidence in oligoene aggregates ${ }^{45,47}$ and conjugated polyenes ${ }^{48}$ has revealed direct triplet formation instead from the bright $1 \mathrm{~B}_{\mathrm{u}}$ state. It thus remains unclear what aspect of oligoene character enables SEF and precisely how the coupling between manifolds is achieved, though it is clear empirically that it is a powerful "ingredient" for SEF materials. ${ }^{26,48}$ Among the reported intramolecular SEF materials, vinylene-containing polymers have typically exhibited some form of optical activation, with fission enhanced by or even requiring excitation with a photon above the bandgap. ${ }^{26,48}$ The lack of intramolecular SEF in shorter oligoenes such as the carotenoids despite suitable energetics suggests that there is also a minimum size requirement for this SEF motif. On the other hand, nonactivated intramolecular singlet fission has been observed in several pentacene and tetracene dimers, with efficiencies as high as $200 \% .^{21-24,28,38,39}$ These results indicate that intramolecular SEF may be possible as long as two distinct sites for the product triplets are available.
The chemical design of CT SEF chromophores can be challenging as it not only involves increasing the $S_{1}-T_{1}$ gap but also endowing the $S_{1}$ state with sufficient CT character to enhance its coupling with the multiexcitonic triplet state. The former involves either the destabilization of the $S_{1}$ state or stabilization of the $T_{1}$ state by fine tuning chromophore structural motifs. The latter is feasible when there is a large electronic coupling between donor and acceptor leading to a large HOMO-LUMO spatial overlap and hence large exchange energies. ${ }^{49}$ The present understanding and the scarcity of appropriately designed materials provide little insight into the possible ways of harvesting multiple excitons in molecular materials. Diketopyrrolopyrrole (DPP)-based polymers and oligomers have attracted attention by virtue of high efficiencies in solar cells $(\sim 10 \%)$ and high hole $\left(\sim 20 \mathrm{~cm}^{2} \mathrm{~V}^{-1} \mathrm{~s}^{-1}\right)$ and electron mobilities $\left(\sim 12 \mathrm{~cm}^{2} \mathrm{~V}^{-1} \mathrm{~s}^{-1}\right)$ in organic field-effect transistors. ${ }^{50,51}$ Their attractive properties such as high electron affinities and relatively low triplet energies can lead to the exploration of novel design principles for next-generation singlet fission materials based on DPP oligomers and polymers. Recent work has suggested that DPP derivatives can undergo SEF in the solid state ${ }^{34}$ thanks in part to the CT character of their excitations, making them strong candidates for intramolecular SEF design motifs. This work addresses the challenge in developing intramolecular singlet fission materials.

In keeping with the aforesaid design principle of combining $\mathrm{CT}$ and polyene character, we coupled together two thiopheneDPP molecules (TDPP) with a vinyl (V) linker to control the interchromophore coupling, yielding the dimer TDPP-VTDPP. UV-vis absorption measurements reveal substantial interaction between the DPP units, while photoluminescence (PL) spectroscopy shows the molecule to be nonemissive, as typically observed in polyenes. Cyclic voltammetry (CV) measurements have been performed to elucidate the ionization potential and electron affinity. In order to probe the excitedstate dynamics and better understand the interplay of bright and dark states, we performed transient absorption (TA) measurements on TDPP-V-TDPP in solution as well as in rigid polystyrene (PS) matrixes. We observe in all cases that the initial "bright" excited state appears to rapidly decay by internal conversion into a dark and extremely short-lived $2 \mathrm{~A}_{\mathrm{g}}$-type singlet, analogous to polyene photophysics because of the $\mathrm{V}$ bridging unit. There is no evidence of triplet or charge formation nor does substantial conformational change drive the rapid excited-state deactivation, which we consider instead to be analogous to the fast vibrationally mediated decay active in carotenoids and other polyenes. Our experiments demonstrate that SEF is inactive in this material, which is likely a consequence of the strongly reduced singlet energy upon dimerization. Our study nonetheless sets important lessons for 
the design principles of DPP-based oligomers for future applications as next-generation SEF materials.

TDPP-V-TDPP was synthesized using a Stille coupling reaction involving a monobromo derivative of DPP and 1,2bis(trisec-butylstannyl)ethene in molar ratios of 2:1 using catalyst $\mathrm{Pd}_{2}(\mathrm{dba})_{3}$ and ligand $\mathrm{P}(\mathrm{o}-\mathrm{tol})_{3}$ in dry toluene as the solvent. The synthesis of TDPP-V-TDPP is shown in Scheme 1. DPP was alkylated with the short hexyl chain to enhance the crystallinity. The intermediates and the final compounds were purified by silica gel chromatography. The monomers and final compounds were characterized, and their purity was verified by ${ }^{1} \mathrm{H}$ NMR and ${ }^{13} \mathrm{C}$ NMR. The spectral data are described in the Supporting Information.

The UV-vis absorption spectra of TDPP-V-TDPP and the corresponding monomer unit are shown in Figure 1. The

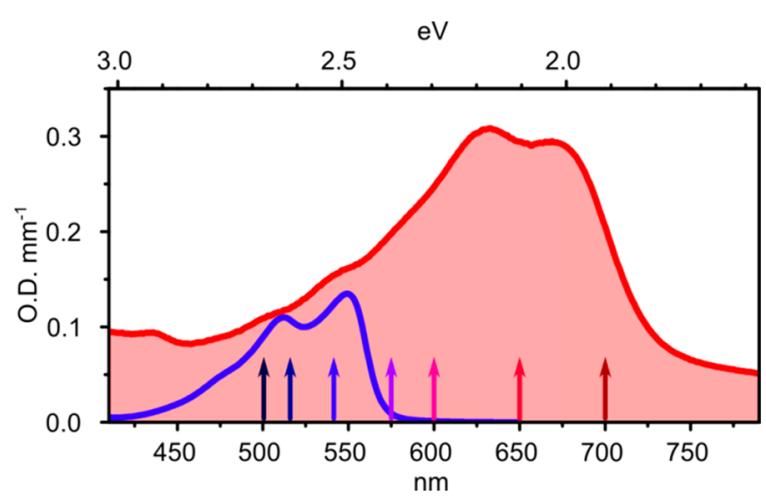

Figure 1. Normalized UV-vis spectrum of TDPP-V-TDPP (red) in chlorobenzene at a concentration of $50 \mu \mathrm{g} / \mathrm{mL}$. The weak features seen at $\sim 500$ and $550 \mathrm{~nm}$ closely match the absorption of the equivalent TDPP monomer (blue, arbitrary scale). The substantial red shift upon dimerization is a signature of very strong interaction between the TDPP units. Arrows indicate pump wavelengths used in TA measurements.

absorption exhibits a substantial red shift of TDPP upon dimerization, from $E_{\mathrm{g}} \approx 2.2$ to $\sim 1.75 \mathrm{eV}$, coincident with a shift in the $0-0 / 0-1$ vibronic peak ratio from 1.2 to 0.95 . These changes are indicative of substantial interaction between TDPP units and demonstrate effective conjugation across the central linker. While interaction between the constituent monomer units is important for processes such as SEF, the very strong effects here may prove detrimental. The triplet energy of the equivalent monomer molecule has been reported to be $\sim 1.1$ $\mathrm{eV},{ }^{34}$ which would result in very unfavorable SEF energetics if unchanged in the dimer. However, this electronic coupling is likely to alter the HOMO-LUMO overlap and consequently the $S_{1}-T_{1}$ gap. In light of this, previous observations of changed triplet energies upon dimerization, ${ }^{24}$ and the typically large exchange energies in polyenes, the red shift of absorption alone is not sufficient to rule out SEF in TDPP-V-TDPP. Interestingly, we also detect slight vibronic features in the range of $500-550 \mathrm{~nm}$, in agreement with the peaks of monomer absorption. We do not anticipate any residual monomer in the solution following purification and detected no signs of such impurities using NMR. We also do not find any evidence for gradual degradation of the TDPP-V-TDPP samples or powders into uncoupled, monomeric molecules. Instead, we attribute these bands to twisted conformers in which the conjugation between TDPP units is minimal. Heating the solution causes a reduction in the $0-0 / 0-1$ vibronic ratio, consistent with increased torsional disorder on average, but the relative weight of these higher-energy peaks does not vary (Figure S4, Supporting Information (SI)). The barrier for interconversion between these states must be substantial, suggesting that these monomer-like bands represent a different stereoisomer that was kinetically trapped in a twisted configuration during synthesis.

We could detect no luminescence from TDPP-V-TDPP, whether in a standard fluorimeter (excitation from 400 to 750 $\mathrm{nm}$ ) or following laser excitation at 550 or $650 \mathrm{~nm}$. We thus conclude that the PL quantum efficiency in the dimer is extremely low due to a short singlet excited-state lifetime. This result suggests similar behavior to the polyenes, where rapid $\left(<200 \mathrm{fs}\right.$ ) deactivation of the bright $1 \mathrm{~B}_{\mathrm{u}}$ state through internal conversion to $2 \mathrm{~A}_{\mathrm{g}}$ results in $\mathrm{PL}$ quantum yields below $10^{-5}$. The cyclic voltammograms are shown in Figure S3, SI. The extracted oxidation and reduction potentials are -5.00 and $-3.44 \mathrm{eV}$, respectively, and the electrochemical gap is $1.56 \mathrm{eV}$. TDPP-V-TDPP exhibits a reversible reduction cycle and a quasi-reversible oxidation cycle.

The low PL efficiency of TDPP-V-TDPP means that other spectroscopic tools are needed to build a comprehensive picture of the excited-state decay processes, namely, TA spectroscopy using a system described previously. ${ }^{48}$ In this technique, the sample is excited with a short, tunable pump pulse, followed at a precisely controllable time delay by a broadband $(490-800$ or $800-1175 \mathrm{~nm}$ ) probe pulse. The transmission spectrum of the probe is measured in the presence and absence of the pump pulse, enabling direct determination of the absorption spectrum of any excited states. Increased transmission of the probe (i.e., $\Delta T / T>0$ ) can be attributed to bleaching of the ground-state absorption or stimulated emission from excited singlet states. A reduction in probe transmission $(\Delta T / T<0)$ gives the unique absorption signature of the excited state. In practice, these features tend to strongly overlap, making identification of a distinct excited state difficult unless there is a clear change in the spectral shape over time or a change in the decay kinetics across the detection bandwidth. Because this technique detects excited-state absorption, it is particularly well suited to materials and states that do not strongly emit, such as TDPP-V-TDPP.

In Figure 2, we present TA spectra $(\mathrm{a}, \mathrm{c})$ and decay kinetics $(\mathrm{b}, \mathrm{d})$ of TDPP-V-TDPP in chlorobenzene solution. Under band-edge excitation (Figure 2a,b), we observe behavior reminiscent of polyenes. The dominant $\Delta T / T>0$ bands closely agree with the absorption spectrum (gray dashed), enabling assignment to ground-state bleach (GSB). Due to the absence of any additional positive features that could be attributed to stimulated emission, we consider the primary species observed (colored traces) to be an optically "dark" state. On the very earliest time scales (thin black line), we detect additional spectral signatures such as a broadened photoinduced absorption (PIA) in the NIR, with distinct absorption of $>1075 \mathrm{~nm}$, and a crossing point between positive and negative TA signals that is shifted $\sim 10 \mathrm{~nm}$ to the red. The presence of this shifted crossing point is a potential sign of stimulated emission of photons from the bright initially excited state, characterized by an extremely short lifetime. These features are assigned as signatures of the initial singlet excited state, which converts on ultrafast time scales to a short-lived dark state, analogous to the rapid $1 \mathrm{~B}_{\mathrm{u}} \rightarrow 2 \mathrm{~A}_{\mathrm{g}}$ internal conversion well-known in the polyenes. The time scale of this process is within our instrument resolution and should be $<200 \mathrm{fs}$; the existence of such rapid internal conversion is 

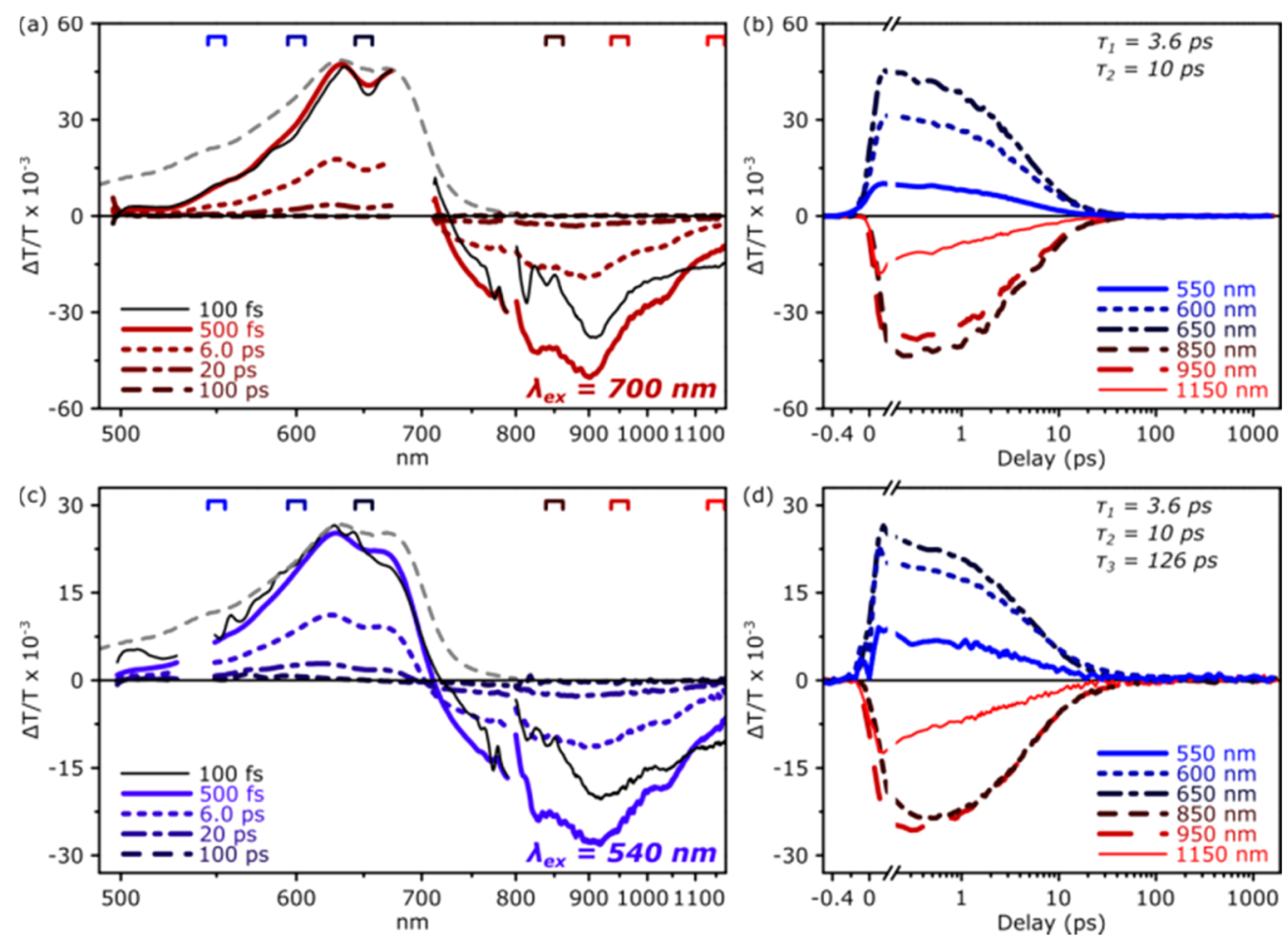

Figure 2. Subpicosecond TA measurements of TDPP-V-TDPP in solution with excitation $(\mathrm{a}, \mathrm{b})$ at the band edge and (c,d) well above the band edge reveal rapid internal conversion and excited-state quenching. (a) The initial excited state (thin black) converts within the instrument response to a second "dark" state, which exhibits no sign of stimulated emission. No further spectral evolution is detected during rapid decay to the ground state. By 100 ps, no signal can be detected. The UV-vis absorption spectrum (gray dashed) shows a close match to the positive TA signals observed, suggesting that none can be attributed to stimulated emission. (b) Decay kinetics integrated over the bands indicated in (a). Following the initial fast kinetics, best observed in the 950 and $1150 \mathrm{~nm}$ traces, the kinetics can be well described with biexponential decay with the indicated time constants. (c) Excitation with significant excess energy results in the same overall spectral behavior but with slight broadening and a change in the shape of the GSB. New long-lived features are apparent, with faint bleach and accompanying PIA detected at 100 ps and beyond. These features agree well with those of TDPP monomers. (d) The decay kinetics following excess energy excitation reveals the same primary decay processes, but an additional decay component must be included in the fit to describe the new long-lived state. A very small fraction of the population persists beyond the limits of the measurement, into the nanosecond regime. Fits are compared to raw data in Figures S6 and S7, SI.
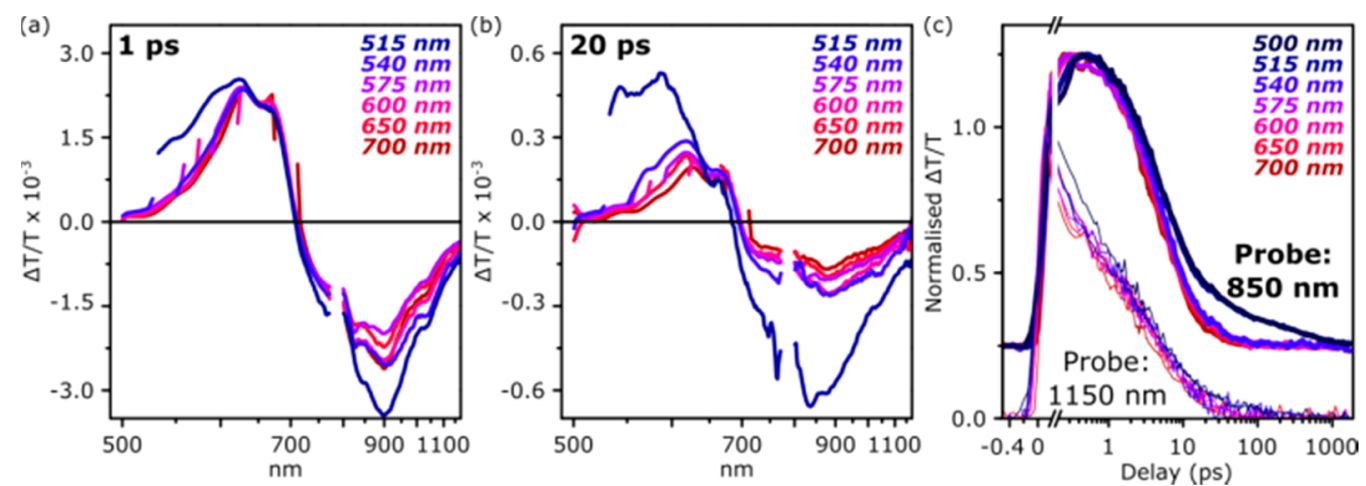

Figure 3. TA measurements at a range of excitation wavelengths reveal systematic spectral changes detectable at (a) early and (b) longer pumpprobe delays. As the pump photon energy is increased, we detect an increase in the PIA signal in the NIR relative to the GSB and a shift in the peak ratio of the GSB spectrum (increasing 0-1 contribution). On longer time scales, the enhancement of PIA and GSB features is still more pronounced as excess photon energy slightly enhances the excited-state lifetime as well. (c) Integrated decay kinetics in the NIR show a small, systematic increase in the excited-state lifetime with pump photon energy until a sharp increase for 515 and $500 \mathrm{~nm}$. This dramatic change is attributed to monomer-like TDPPs, whether arising from a small fraction of uncoupled precursors or from particular dimer conformations in which the TDPP units are not well conjugated. The smaller, systematic changes in the lifetime and TA spectra (particularly the GSB) reflect the pump wavelength selectivity of particular subpopulations of TDPP-V-TDPP with varying conjugation length (i.e., torsional disorder). Full data are presented in Figure S8, SI.

consistent with the absence of detectable fluorescence. The lifetime of the second state is well described with biexponential decay, with the primary decay attributed to the 3.6 ps component. There is no evidence of changing spectral shape over the lifetime of this state, suggesting that the two time constants do not reflect internal conversion between different excited states.

Similar basic features are evident following excitation with excess pump photon energy, as at $540 \mathrm{~nm}$ (Figure 2c,d). The same signatures of initial (thin black) and longer-lived (blue) 

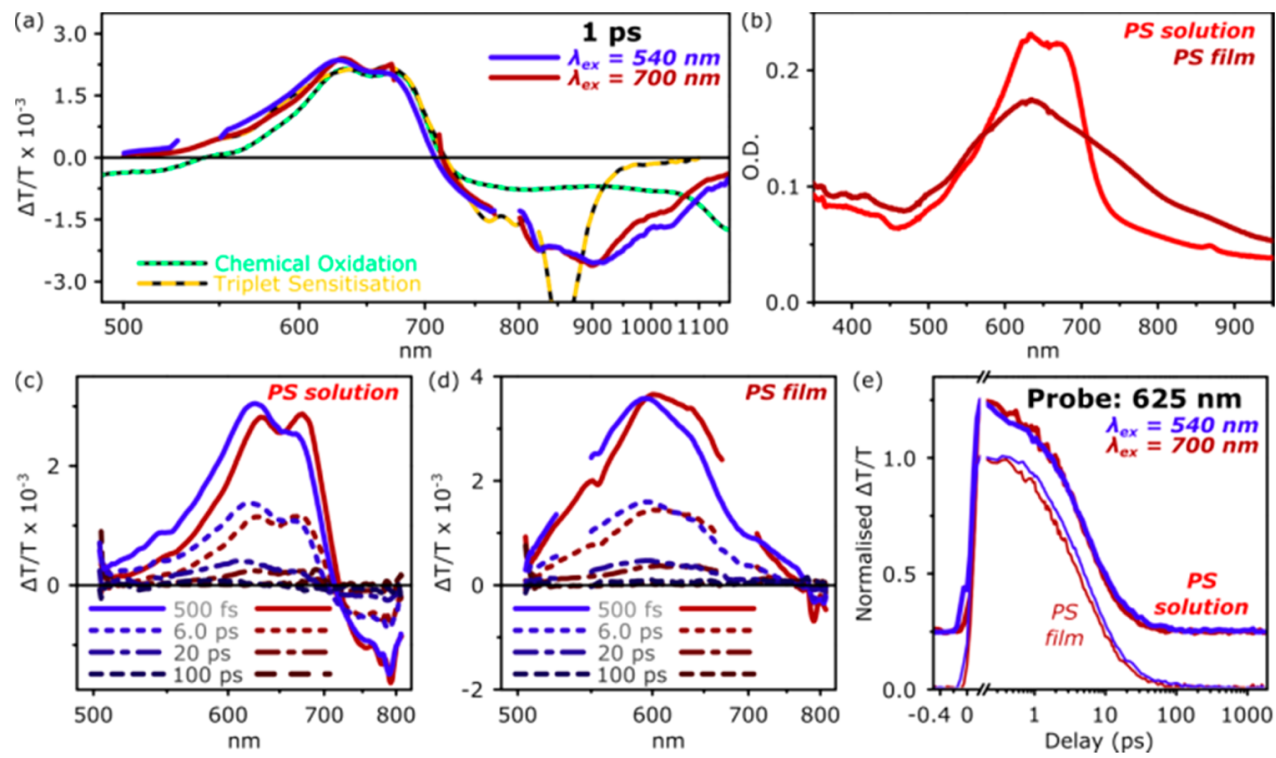

Figure 4. A series of control measurements were performed to identify the source of the ultrafast dynamics in TDPP-V-TDPP. (a) Chemical oxidation and triplet sensitization spectra reveal no similarity to the observed TA spectra following direct excitation nor to the difference between band-edge and excess-energy excitation. There is thus no evidence for SEF or ultrafast charge formation. Full sensitization data are presented in Figure S9, SI. (b) UV-vis absorption spectra of TDPP-V-TDPP in a high-viscosity solution and rigid polymer matrix. (c) TA spectra in concentrated PS solution are essentially indistinguishable from those obtained in chlorobenzene solutions, following both band-edge (dark red, $700 \mathrm{~nm}$ ) and excess-energy (blue, $540 \mathrm{~nm}$ ) excitation. (d) TA spectra in a PS matrix reveal the same qualitative differences between excitation at 540 (blue) and $700 \mathrm{~nm}$ (dark red). (e) Integrated decay kinetics at the GSB peak reveal the same behavior in a viscous solution (thick) and PS matrix (thin) as that previously observed in chlorobenzene solutions, a slight enhancement of the lifetime following excess-energy excitation (blue), with the decay dominated by the same 3.6 and 10 ps time constants.

states can be observed, with only a slight difference in the shape of the GSB. However, on the longest time scales (100 ps and beyond), we can distinguish a faint GSB band at $600 \mathrm{~nm}$ and corresponding PIA at $700-800 \mathrm{~nm}$. These are signatures of a new longer-lived state. We find that an additional exponential component (126 ps) is needed to describe the decay dynamics, and a very small fraction of the population persists beyond the 1.5 ns range of our measurements. Such effects could be consistent with optically activated singlet fission, as has been observed in other polyenes, or the activation of other decay channels through excess energy excitation. To clearly establish the nature of this behavior, we systematically measured the pump wavelength dependence.

As shown in Figure 3, we detect qualitatively similar spectral effects of increased pump photon energy at both (a) early and (b) long pump-probe delays. The primary effects are an increase in the relative weight of the PIA in the NIR and a change in the vibronic peak ratio in the GSB, with a progressive increase and broadening of the $0-1$ peak.

These changes are particularly pronounced on longer time scales as increased pump photon energy also increases the excited-state lifetime. In addition to this subtle but systematic effect in the decay kinetics (Figure 3c), we find a sharp change in behavior for the two highest-energy pump wavelengths (500 and $515 \mathrm{~nm}$ ). At these wavelengths, a substantial amount of the longer-lived excited state is generated. We find no evidence, however, for assignment to optically activated SEF as the triplet spectrum obtained through an established sensitization method using $N$-methylfulleropyrrolidine as a triplet donor ${ }^{48}$ exhibits markedly different PIA (Figure 4a). Likewise, it is clear that this longer-lived species is not a CT state as there is a poor match to the spectrum from chemical doping (Figure 4a). The match of all spectra at the short-wavelength edge of the excited-state absorption is intriguing. A similar shoulder at $\sim 700-800 \mathrm{~nm}$ is seen under all direct excitation conditions, as well as in the sensitized triplet spectrum and following chemical oxidation, albeit with much lower intensity relative to the GSB. While the onset of this band is largely governed by the position of the ground-state absorption, the similarity of the shoulder suggests a more fundamental relationship between the origin of the bands. We propose that the reason lies in the underlying CT character of excitations in DPP materials in general. This shoulder is evident in polaronic states (chemical reduction) and may be taken as a signature of CT character. Mixing of $S_{1}$ and $\mathrm{T}_{1}$ states with CT configurations may then endow the PIA with such signatures, analogous to the singlet-triplet mixed features reported in bound ${ }^{1}(\mathrm{TT})$ states in TIPS-tetracene solutions. ${ }^{52}$

Comparison of the long-lived spectra following excitation at $<600 \mathrm{~nm}$ (Figures S4 and S5, SI) with published data reveals a striking similarity to the bright singlet state in a monomer TDPP derivative in solution. ${ }^{34}$ Indeed, we find that the largest effects in the pump wavelength dependence can all be accounted for as selective excitation at high pump photon energies of a subpopulation of monomer-like molecules. As discussed above in the context of the absorption spectrum, we expect that these molecules are not chemical monomers but rather conformers in which the conjugation between TDPP units is ineffective. We find that selectivity of different degrees of torsional disorder can explain the more subtle, systematic changes in TA spectra and kinetics observed across the entire pump wavelength series. Lower pump photon energies selectively excite molecules with more extended conjugation and presumably more planalized structure, as reflected in the $0-0 / 0-1$ vibronic ratio observed in the GSB (a direct measure of the "type" of molecules excited). Molecules in such geometry are likely closer to the equilibrium excited-state geometry and 
thus have a greater radiative rate and need less time for vibrational relaxation, resulting in a slightly shorter excited-state lifetime. The pump wavelength dependence does not explain the surprisingly short primary excited-state lifetime, which is well described by the same 3.6 and 10 ps time constants in all cases (discounting the effects of the monomer-like species). As one of the most likely decay channels for such a covalent dimer is nonradiative decay via torsional relaxation about the central bond, ${ }^{53,54}$ we investigated the effects of geometric constraint in high-viscosity PS solution ( $95 \mathrm{mg} / \mathrm{mL}$ in chlorobenzene) and a rigid PS matrix (Figure $4 \mathrm{~b}-\mathrm{e}$ ).

In PS solution, we observe the same absorption line shape as that in dilute chlorobenzene, while the spin-cast PS matrix exhibits pronounced broadening and a red shift. These changes could suggest the presence of intermolecular interactions despite the relatively low TDPP-V-TDPP loading (50 $\mu \mathrm{g}$ / $\mathrm{mL}$ ). However, there is no corresponding change in the excited-state dynamics. We propose instead that the broadened and strongly red shifted spectrum reflects the planarizing effects of the PS matrix, resulting in some molecules with significantly enhanced conjugation length. In viscous solution (Figure 4c), we observe the same spectral shapes and same pump wavelength dependence identified previously: an excitationdependent vibronic structure in the GSB, no sign of stimulated emission and a PIA toward the NIR. While the TA spectra of the polymer matrix (Figure 4d) appear very different in shape, comparison with the absorption spectrum confirms that the entire spectral shape is consistent with GSB. There are no new features corresponding to the longer-lived excited states or a change in decay pathway due to conformational restriction. In fact, the overall behavior appears to be entirely consistent with measurements in "free" solution; as previously, excitation with higher-energy photons results in an enhancement of the GSB on the "blue" side and a reduction on the "red" side, pointing to the same selectivity of torsional conformers.

In no case do we observe a significant change in the excitedstate lifetime (Figure 4e). The decay in PS solution and even PS matrix is almost indistinguishable from that in chlorobenzene solution, ruling out fast large-scale conformational change as the primary decay pathway. We thus consider that the photophysics observed here are most consistent with purely electronic dynamics, following the polyene model in which ultrafast internal conversion from the initial bright state forms a dark state of $A_{g}$ symmetry. This state in turn exhibits very strong coupling to the ground-state vibrational manifold and rapidly decays through multiphonon emission.

Our chemical strategy for imbuing polyene character in a donor-acceptor-based covalent dimer appears to be successful but results in no singlet fission. We expect that the primary reason for this behavior lies in the energy balance. Dimerization into TDPP-V-TDPP results in a significant stabilization of the singlet energy, by $\sim 400 \mathrm{meV}$. It is evident that there is no comparable reduction in the triplet energy, which is estimated at $1.1 \mathrm{eV}$ in the monomer; the net result of this would be that triplet pair formation is energetically inaccessible. Instead, inclusion of the $\mathrm{V}$ linker creates the possibility of a dark, lowerenergy state analogous to the $2 \mathrm{~A}_{\mathrm{g}}$ state analogous to polyenes, which decays rapidly and nonradiatively back to the ground state. This surprising effect reveals some of the possible pitfalls of small structural changes to design SEF dimers. However, the results also demonstrate that combining polyene character with CT chromophores can be straightforwardly achieved, and the features observed in the PIA spectra suggest that the CT character is maintained in the excited states. The remaining challenge, then, is to modulate the exchange energy to make SEF energetically accessible without too significantly perturbing the beneficial CT mixing. Destabilization of the $S_{1}$ state should reduce the of $S_{1}$ and CT states while simultaneously increasing the $S_{1}-T_{1}$ gap. ${ }^{26,49}$ This can be modulated by incorporating a covalent linker to control the extent of HOMO-LUMO spatial overlap in the chromophore. Rational choice or design of covalent linkers such as phenyls, biphenyls, ${ }^{55}$ or their substituted analogues can increase the energy of the $S_{1}$ state by steric or hyperconjugation effects. End-capping the oligomer with strong electron-withdrawing groups such as cyano and so forth will increase the proportion of quinoidal forms and form biradicals that stabilize the triplet state. $^{56}$ Such synthetic strategies are currently being implemented in our laboratory, and spectroscopic studies are underway.

\section{ASSOCIATED CONTENT}

\section{Supporting Information}

The Supporting Information is available free of charge on the ACS Publications website at DOI: 10.1021/acs.jpclett.6b02919. Data availability: The data underlying this publication are available at http://10.15131/shef.data.4640731.

Materials and methods, Synthetic procedures, ${ }^{1} \mathrm{H}$ NMR,

${ }^{13} \mathrm{C} \mathrm{NMR}$, cyclic voltammograms, UV-vis after doping, full transient absorption data and fitting, and triplet sensitization (Figures S1-S9) (PDF)

\section{AUTHOR INFORMATION}

\section{Corresponding Authors}

*E-mail: satish@sscu.iisc.ernet.in (S.P.).

*E-mail: a.musser@sheffield.ac.uk (A.J.M.).

ORCID

Andrew J. Musser: 0000-0002-4600-6606

Author Contributions

§ T.M. and A.J.M. contributed equally.

Notes

The authors declare no competing financial interest.

\section{ACKNOWLEDGMENTS}

We thank the NMR Research Centre, Indian Institute of Science, Bangalore for the NMR facility. S.P., R.H.F., and A.J.M. thank UKIERI, British Council for a research grant. T.M. thanks IISc for a Senior Research Fellowship. This work was supported by the Engineering and Physical Sciences Research Council, U.K. (Grant Numbers EP/M005143/1, EP/ G060738/1, and EP/M025330/1: "Hybrid Polaritonics”).

\section{REFERENCES}

(1) Kawashima, K.; Tamai, Y.; Ohkita, H.; Osaka, I.; Takimiya, K. High-efficiency polymer solar cells with small photon energy loss. Nat. Commun. 2015, 6, 10085.

(2) Kim, J.-H.; Park, J. B.; Yang, H.; Jung, I. H.; Yoon, S. C.; Kim, D.; Hwang, D.-H. Controlling the Morphology of BDTT-DPP-Based Small Molecules via End-Group Functionalization for Highly Efficient Single and Tandem Organic Photovoltaic Cells. ACS Appl. Mater. Interfaces 2015, 7, 23866-23875.

(3) Li, W.; Hendriks, K. H.; Wienk, M. M.; Janssen, R. A. J. Diketopyrrolopyrrole Polymers for Organic Solar Cells. Acc. Chem. Res. 2016, 49, 78-85.

(4) Liu, F.; Gu, Y.; Wang, C.; Zhao, W.; Chen, D.; Briseno, A. L.; Russell, T. P. Efficient Polymer Solar Cells Based on a Low Bandgap 
Semi-crystalline DPP Polymer-PCBM Blends. Adv. Mater. 2012, 24, 3947-3951.

(5) Zhang, S.; Ye, L.; Hou, J. Breaking the 10\% Efficiency Barrier in Organic Photovoltaics: Morphology and Device Optimization of WellKnown PBDTTT Polymers. Adv. Energy Mater. 2016, 6, 1502529.

(6) Son, H. J.; Carsten, B.; Jung, I. H.; Yu, L. Overcoming efficiency challenges in organic solar cells: rational development of conjugated polymers. Energy Environ. Sci. 2012, 5, 8158-8170.

(7) Xu, Y.; Gong, T.; Munday, J. N. The generalized ShockleyQueisser limit for nanostructuredsolar cells. Sci. Rep. 2015, 5, 13536.

(8) Nelson, C. A.; Monahan, N. R.; Zhu, X. Y. Exceeding the Shockley-Queisser limit in solar energy conversion. Energy Environ. Sci. 2013, 6, 3508-3519.

(9) Wu, T. C.; Thompson, N. J.; Congreve, D. N.; Hontz, E.; Yost, S. R.; Van Voorhis, T.; Baldo, M. A. Singlet fission efficiency in tetracenebased organic solar cells. Appl. Phys. Lett. 2014, 104, 193901.

(10) Smith, M. B.; Michl, J. Singlet Fission. Chem. Rev. 2010, 110, 6891-6936.

(11) Zhang, Y.-D.; Wu, Y.; Xu, Y.; Wang, Q.; Liu, K.; Chen, J.-W.; Cao, J.-J.; Zhang, C.; Fu, H.; Zhang, H.-L. Excessive Exoergicity Reduces Singlet Exciton Fission Efficiency of Heteroacenes in Solutions. J. Am. Chem. Soc. 2016, 138, 6739-6745.

(12) Hanna, M. C.; Nozik, A. J. Solar conversion efficiency of photovoltaic and photoelectrolysis cells with carrier multiplication absorbers. J. Appl. Phys. 2006, 100, 074510.

(13) Zimmerman, P. M.; Zhang, Z.; Musgrave, C. B. Singlet fission in pentacene through multi-exciton quantum states. Nat. Chem. 2010, 2, 648-652.

(14) Kato, D.; Sakai, H.; Tkachenko, N. V.; Hasobe, T. High-Yield Excited Triplet States in Pentacene Self-Assembled Monolayers on Gold Nanoparticles through Singlet Exciton Fission. Angew. Chem., Int. Ed. 2016, 55, 5230-5234.

(15) Herz, J.; Buckup, T.; Paulus, F.; Engelhart, J.; Bunz, U. H. F.; Motzkus, M. Acceleration of Singlet Fission in an Aza-Derivative of TIPS-Pentacene. J. Phys. Chem. Lett. 2014, 5, 2425-2430.

(16) Wilson, M. W. B.; Rao, A.; Clark, J.; Kumar, R. S. S.; Brida, D.; Cerullo, G.; Friend, R. H. Ultrafast Dynamics of Exciton Fission in Polycrystalline Pentacene. J. Am. Chem. Soc. 2011, 133, 11830-11833.

(17) Wilson, M. W. B.; Rao, A.; Ehrler, B.; Friend, R. H. Singlet Exciton Fission in Polycrystalline Pentacene: From Photophysics toward Devices. Acc. Chem. Res. 2013, 46, 1330-1338.

(18) Yost, S. R.; Lee, J.; Wilson, M. W. B.; Wu, T.; McMahon, D. P.; Parkhurst, R. R.; Thompson, N. J.; Congreve, D. N.; Rao, A.; Johnson, K.; Sfeir, M. Y.; Bawendi, M. G.; Swager, T. M.; Friend, R. H.; Baldo, M. A.; Van Voorhis, T. A transferable model for singlet-fission kinetics. Nat. Chem. 2014, 6, 492-497.

(19) Musser, A. J.; Liebel, M.; Schnedermann, C.; Wende, T.; Kehoe, T. B.; Rao, A.; Kukura, P. Evidence for conical intersection dynamics mediating ultrafast singlet exciton fission. Nat. Phys. 2015, 11, 352357.

(20) Feng, X.; Casanova, D.; Krylov, A. I. Intra- and Intermolecular Singlet Fission in Covalently Linked Dimers. J. Phys. Chem. C 2016, 120, 19070.

(21) Zirzlmeier, J.; Casillas, R.; Reddy, S. R.; Coto, P. B.; Lehnherr, D.; Chernick, E. T.; Papadopoulos, I.; Thoss, M.; Tykwinski, R. R.; Guldi, D. M. Solution-based intramolecular singlet fission in crossconjugated pentacene dimers. Nanoscale 2016, 8, 10113-10123.

(22) Fuemmeler, E. G.; Sanders, S. N.; Pun, A. B.; Kumarasamy, E.; Zeng, T.; Miyata, K.; Steigerwald, M. L.; Zhu, X. Y.; Sfeir, M. Y.; Campos, L. M.; Ananth, N. A Direct Mechanism of Ultrafast Intramolecular Singlet Fission in Pentacene Dimers. ACS Cent. Sci. 2016, 2, 316-324.

(23) Sanders, S. N.; Kumarasamy, E.; Pun, A. B.; Steigerwald, M. L.; Sfeir, M. Y.; Campos, L. M. Intramolecular Singlet Fission in Oligoacene Heterodimers. Angew. Chem., Int. Ed. 2016, 55, 33733377.

(24) Lukman, S.; Musser, A. J.; Chen, K.; Athanasopoulos, S.; Yong, C. K.; Zeng, Z.; Ye, Q.; Chi, C.; Hodgkiss, J. M.; Wu, J.; Friend, R. H.; Greenham, N. C. Tuneable Singlet Exciton Fission and Triplet-
Triplet Annihilation in an Orthogonal Pentacene Dimer. Adv. Funct. Mater. 2015, 25, 5452-5461.

(25) Damrauer, N. H.; Snyder, J. L. Symmetry-Directed Control of Electronic Coupling for Singlet Fission in Covalent Bis-Acene Dimers. J. Phys. Chem. Lett. 2015, 6, 4456-4462.

(26) Busby, E.; Xia, J.; Wu, Q.; Low, J. Z.; Song, R.; Miller, J. R.; Zhu, X. Y.; Campos, L. M.; Sfeir, M. Y. A design strategy for intramolecular singlet fission mediated by charge-transfer states in donor-acceptor organic materials. Nat. Mater. 2015, 14, 426-433.

(27) Beljonne, D.; Yamagata, H.; Brédas, J. L.; Spano, F. C.; Olivier, Y. Charge-Transfer Excitations Steer the Davydov Splitting and Mediate Singlet Exciton Fission in Pentacene. Phys. Rev. Lett. 2013, 110, 226402.

(28) Chan, W.-L.; Berkelbach, T. C.; Provorse, M. R.; Monahan, N. R.; Tritsch, J. R.; Hybertsen, M. S.; Reichman, D. R.; Gao, J.; Zhu, X. Y. The Quantum Coherent Mechanism for Singlet Fission: Experiment and Theory. Acc. Chem. Res. 2013, 46, 1321-1329.

(29) Berkelbach, T. C.; Hybertsen, M. S.; Reichman, D. R. Microscopic theory of singlet exciton fission. III. Crystalline pentacene. J. Chem. Phys. 2014, 141, 074705.

(30) Berkelbach, T. C.; Hybertsen, M. S.; Reichman, D. R. Microscopic theory of singlet exciton fission. I. General formulation. J. Chem. Phys. 2013, 138, 114102.

(31) Ito, S.; Nagami, T.; Nakano, M. Design Principles of Electronic Couplings for Intramolecular Singlet Fission in Covalently-Linked Systems. J. Phys. Chem. A 2016, 120, 6236-6241.

(32) Pensack, R. D.; Ostroumov, E. E.; Tilley, A. J.; Mazza, S.; Grieco, C.; Thorley, K. J.; Asbury, J. B.; Seferos, D. S.; Anthony, J. E.; Scholes, G. D. Observation of Two Triplet-Pair Intermediates in Singlet Exciton Fission. J. Phys. Chem. Lett. 2016, 7, 2370-2375.

(33) Smith, M. B.; Michl, J. Recent Advances in Singlet Fission. Annu. Rev. Phys. Chem. 2013, 64, 361-386.

(34) Hartnett, P. E.; Margulies, E. A.; Mauck, C. M.; Miller, S. A.; Wu, Y.; Wu, Y.-L.; Marks, T. J.; Wasielewski, M. R. Effects of Crystal Morphology on Singlet Exciton Fission in Diketopyrrolopyrrole Thin Films. J. Phys. Chem. B 2016, 120, 1357-1366.

(35) Margulies, E. A.; Miller, C. E.; Wu, Y.; Ma, L.; Schatz, G. C.; Young, R. M.; Wasielewski, M. R. Enabling singlet fission by controlling intramolecular charge transfer in $\pi$-stacked covalent terrylenediimide dimers. Nat. Chem. 2016, 8, 1120.

(36) Nakano, M. Open-Shell-Character-Based Molecular Design Principles: Applications to Nonlinear Optics and Singlet Fission. Chem. Rec. 2016, 3236-3256.

(37) Pensack, R. D.; Tilley, A. J.; Parkin, S. R.; Lee, T. S.; Payne, M. M.; Gao, D.; Jahnke, A. A.; Oblinsky, D. G.; Li, P.-F.; Anthony, J. E.; Seferos, D. S.; Scholes, G. D. Exciton Delocalization Drives Rapid Singlet Fission in Nanoparticles of Acene Derivatives. J. Am. Chem. Soc. 2015, 137, 6790-6803.

(38) Korovina, N. V.; Das, S.; Nett, Z.; Feng, X.; Joy, J.; Haiges, R; Krylov, A. I.; Bradforth, S. E.; Thompson, M. E. Singlet Fission in a Covalently Linked Cofacial Alkynyltetracene Dimer. J. Am. Chem. Soc. 2016, 138, 617-627.

(39) Sanders, S. N.; Kumarasamy, E.; Pun, A. B.; Appavoo, K.; Steigerwald, M. L.; Campos, L. M.; Sfeir, M. Y. Exciton Correlations in Intramolecular Singlet Fission. J. Am. Chem. Soc. 2016, 138, 72897297.

(40) Zeng, T.; Goel, P. Design of Small Intramolecular Singlet Fission Chromophores: An Azaborine Candidate and General Small Size Effects. J. Phys. Chem. Lett. 2016, 7, 1351-1358.

(41) Monahan, N.; Zhu, X.-Y. Charge Transfer-Mediated Singlet Fission. Annu. Rev. Phys. Chem. 2015, 66, 601-618.

(42) Mirjani, F.; Renaud, N.; Gorczak, N.; Grozema, F. C. Theoretical Investigation of Singlet Fission in Molecular Dimers: The Role of Charge Transfer States and Quantum Interference. J. Phys. Chem. C 2014, 118, 14192-14199.

(43) Busby, E.; Xia, J.; Low, J. Z.; Wu, Q.; Hoy, J.; Campos, L. M.; Sfeir, M. Y. Fast Singlet Decay in Push-Pull Molecules Contained Oxidized Thiophenes. J. Phys. Chem. B 2015, 119, 7644-7650. 
(44) Aryanpour, K.; Shukla, A.; Mazumdar, S. Theory of Singlet Fission in Polyenes, Acene Crystals, and Covalently Linked Acene Dimers. J. Phys. Chem. C 2015, 119, 6966-6979.

(45) Musser, A. J.; Maiuri, M.; Brida, D.; Cerullo, G.; Friend, R. H.; Clark, J. The Nature of Singlet Exciton Fission in Carotenoid Aggregates. J. Am. Chem. Soc. 2015, 137, 5130-5139.

(46) Tavan, P.; Schulten, K. Electronic excitations in finite and infinite polyenes. Phys. Rev. B: Condens. Matter Mater. Phys. 1987, 36, 4337-4358.

(47) Wang, C.; Tauber, M. J. High-Yield Singlet Fission in a Zeaxanthin Aggregate Observed by Picosecond Resonance Raman Spectroscopy. J. Am. Chem. Soc. 2010, 132, 13988-13991.

(48) Musser, A. J.; Al-Hashimi, M.; Maiuri, M.; Brida, D.; Heeney, M.; Cerullo, G.; Friend, R. H.; Clark, J. Activated Singlet Exciton Fission in a Semiconducting Polymer. J. Am. Chem. Soc. 2013, 135, 12747-12754.

(49) Chen, T.; Zheng, L.; Yuan, L.; An, Z.; Chen, R.; Tao, Y.; Li, H.; Xie, X.; Huang, W. Understanding the control of Singlet-Triplet Splitting for Organic Exciton Manipulating: A combined Theoritical and Experimental Approach. Sci. Rep. 2015, 5, 10923.

(50) Kang, I.; Yun, H.-J.; Chung, D. S.; Kwon, S.-K.; Kim, Y.-H. Record High Hole Mobility in Polymer Semiconductors via SideChain Engineering. J. Am. Chem. Soc. 2013, 135, 14896-14899.

(51) Sun, B.; Hong, W.; Yan, Z.; Aziz, H.; Li, Y. Record High Electron Mobility of $6.3 \mathrm{~cm} 2 \mathrm{~V}-1 \mathrm{~s}-1$ Achieved for Polymer Semiconductors Using a New Building Block. Adv. Mater. 2014, 26, 2636-2642.

(52) Stern, H. L.; Musser, A. J.; Gelinas, S.; Parkinson, P.; Herz, L. M.; Bruzek, M. J.; Anthony, J.; Friend, R. H.; Walker, B. J. Identification of a Triplet Pair Intermediate in Singlet Fission in Solution. Proc. Natl. Acad. Sci. U. S. A. 2015, 112, 7656-7661.

(53) Hayashi, Y.; Yamaguchi, S.; Cha, W. Y.; Kim, D.; Shinokubo, H. Synthesis of Directly Connected BODIPY Oligomers through SuzukiMiyaura Coupling. Org. Lett. 2011, 13, 2992-2995.

(54) Li, F.; Yang, S. I.; Ciringh, Y.; Seth, J.; Martin, C. H.; Singh, D. L.; Kim, D.; Birge, R. R.; Bocian, D. F.; Holten, D.; Lindsey, J. S. Design, Synthesis, and Photodynamics of Light-Harvesting Arrays Comprised of a Porphyrin and One, Two, or Eight Boron-Dipyrrin Accessory Pigments. J. Am. Chem. Soc. 1998, 120, 10001-10017.

(55) Muller, A. M.; Avlasevich, Y. S.; Schoeller, W. W.; Mullen, K.; Bardeen, C. J. Exciton Fission and Fusion in Bis(Tetracene) Molecules with Different Covalent Linker Structures. J. Am. Chem. Soc. 2007, 129, 14240-14250.

(56) Chien, A. D.; Molina, A. R.; Abeyasinghe, N.; Varnavski, O. P.; Goodson, T.; Zimmerman, P. M. Structure and Dynamics of the ${ }^{1}$ (TT) State in a Quinoidal Bithiophene: Characterizing a Promising Intramolecular Singlet Fission Candidate. J. Phys. Chem. C 2015, 119, 28258-28268. 